\title{
The ins and outs of conical refraction
}

James G. Lunney and Denis Weaire,

School of Physics, Trinity College Dublin • Dublin $2 \cdot$ Ireland

T $\mathrm{t}$ was the Irish poet Aubrey de Vere (1814-1902), flushed with patriotic pride, who called conical refraction "the radiant stranger" in a florid passage in his memoirs. The two physicists responsible for the theoretical prediction and experimental confirmation of conical refraction were William Rowan Hamilton (1805-1865) and Humphrey Lloyd (1800-1881), both professors of Trinity College Dublin (fig. 1). Ireland's recent Year of Hamilton (2005) provided an opportunity for re-examining and celebrating their achievement.

Stripped to their essentials, two versions of conical refraction are shown in figure 2, adapted from Thomas Preston's Theory of Light (1890) [1]. In both cases a crystal causes a narrow beam of light to develop into a hollow cone, where a pair of rays might be expected from the theory of double refraction.

Unusually in science, other researchers do not seem to have had any vague premonition of the curious effect before Hamilton's insight and formal theory exposed it. By 1832 Fresnel's wave theory of light had become one of the most worked-over topics in physics, yet an important detail had escaped attention in both theory and experiment. Perhaps it is more excusable to overlook the effect in experiment since it requires a crystal that is both biaxial and of good optical quality. Moreover the effect is a small one in practice: the cones in figure 2 normally have angles of only a few degrees.

As for theory, many of Hamilton's contemporaries must have felt disappointed that they had failed to notice the anomaly. A Trinity colleague, James MacCullagh (1809-1847), was distraught to the point of launching a pointless retrospective campaign for credit. That failure, and his general eclipse by Hamilton, may have contributed to the eventual suicide of MacCullagh in 1847. Fresnel (1788-1827) did not live quite long enough to suffer any pangs of remorse at his oversight.

For Hamilton it was a crowning achievement, a realisation of his precocious promise [2]. For mathematical physics in general it was a significant milestone: arguably the first mathematical prediction of a novel physical property that was subsequently confirmed by experiment. It has been said that Hamilton claimed that his theory was so secure that it had no need of experimental validation. If he did say this, it must have been a rare jest from this serious man, for he did not regard the theory as a closed book. He did everything he could to encourage and assist Lloyd in his difficult task.

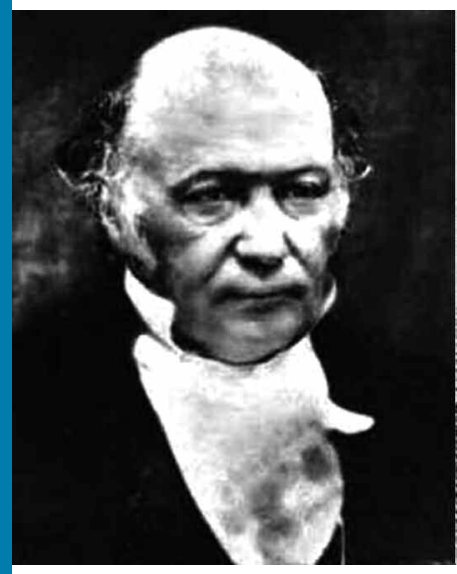

(a)

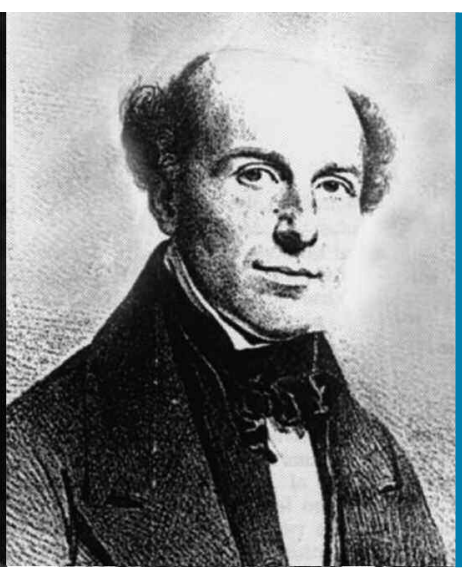

(b)
Isaac Todhunter (1820-1884) once made a jocular remark that, having taught this subject all his life he did not want to have his ideas upset by a demonstration. Those ideas might well have been upset by some of what follows below.

Whether the conical refraction story was a triumph for the wave theory of light (as distinct from those theories based on particles) was debated at the time, but Hamilton's success certainly added momentum to its growing acceptance. The discovery was no paradigm shift, despite being totally unexpected. It was a confirmation of a growing orthodoxy.

James O'Hara, in his 1982 telling of the story, wrote that "it was little more than a curious optical phenomenon which had no conceivable application" [3]. After being highlighted in some of the optical textbooks of the $19^{\text {th }}$ century, conical refraction had indeed been consigned to the lumber-room of miscellaneous minor curiosities. Preston's compendious work included it, but with no great drama. At about the same time Fletcher seems to have completely ignored it in his otherwise exhaustive treatment of double refraction, The Optical Indicatrix and the Transmission of Light in Crystals (1892).

However the topic did catch the attention of Raman in the 1940s when he investigated conical refraction in crystalline naphthalene and made an important contribution to its understanding [4].

But lately conical refraction has been taken out and dusted off. Like most antique curiosities in physics, it contains further layers of intriguing detail if closely examined. Berry has pointed out that conical refraction is the first example of a wave singularity to be discussed in the scientific literature [5]. And in the age of lasers and optical communication the search is on for novel applications. A manufacturer of crystal optics, Vision Crystal Technology AG, is marketing a laser beam shaping device based on internal conical refraction. It seems likely that new applications will emerge to exploit the unique spatial distribution of intensity and polarisation which can be produced by conical refraction.

\section{Essential Theory}

Whether pursued with algebra (as Hamilton did) or with geometry (as many physicists would prefer), an understanding of conical refraction requires an extensive background of optical theory. Its literature, old and new, is obscured by an extraordinary variety and ambiguity of basic nomenclature that is not easily assimilated. So we will try to offer at least a friendly introduction.

From today's perspective, the heart of the matter is Maxwell's theory, applied to the propagation of light in a transparent (nonmagnetic) medium, in our case a crystal. In general it allows a light wave to take the familiar plane-wave form, with wave-vector $\mathbf{k}$, and field amplitudes E, D, B, H, just like a light wave in a vacuum, except for one significant difference. While $\mathbf{D}, \mathbf{B}$, and $\mathbf{H}$ are always to be oriented as in the vacuum, $\mathbf{E}$ can be canted at an angle, while remaining in the plane of $\mathbf{D}$ and $\mathbf{k}$, as shown in figure 3 . Maxwell allows this freedom, and no more.

For a given crystal, $\mathbf{E}$ is a function of $\mathbf{D}$ that is dictated by its dielectric polarisation properties. It is usually a very good approximation to assume that this is a linear function, but this does not 

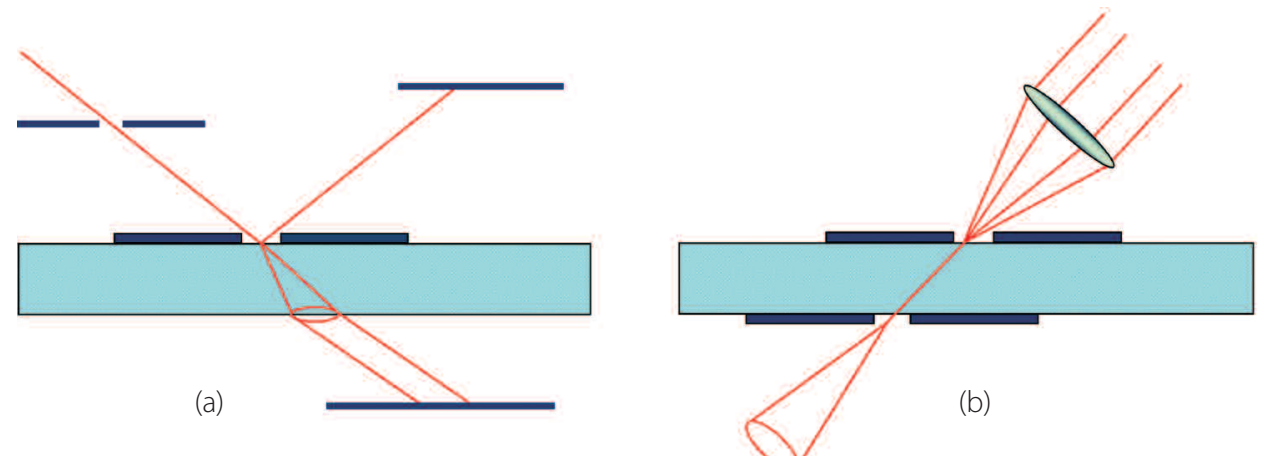

imply that the vectors $\mathbf{D}$ and $\mathbf{E}$ must point in the same direction. Except for cubic crystals and other isotropic materials, they do not.

So to find acceptable light waves for any direction of $\mathbf{k}$, we must find directions of $\mathbf{D}$ (at right angles to $\mathbf{k}$ ) such that $\mathbf{E}$ lies in the required plane. In a crystal of low symmetry we find two distinct directions for $\mathbf{D}$ and can proceed to construct two waves of different polarisation. Maxwell's theory then specifies the velocities for these waves.

Much of optics is described in terms of rays rather than plane waves. A ray is the path taken by a narrow beam of light: it travels in the direction of the vector $\mathbf{S}$ that Poynting introduced into Maxwell's theory. Just as we cannot assume that $\mathbf{E}$ is parallel to $\mathbf{D}$ in a crystal, neither can we assume that $\mathbf{S}$ is parallel to $\mathbf{k}$. Rather, it is perpendicular to $\mathbf{E}$ and $\mathbf{H}$, whereas $\mathbf{k}$ is perpendicular to $\mathbf{D}$ and $\mathbf{H}$.

So a correspondence exists between the directions of rays and waves, but not a simple one in the case of a crystal of low symmetry. In general, two rays are associated with a wave of given direction, and vice-versa. Whenever rays are refracted, we may revert to consideration of the corresponding waves, for refraction is a wave property: incident and refracted waves must match at an interface.

The traditional geometrical representation of crystal optics proceeds from the optical indicatrix, expressing the $\mathbf{E}(\mathbf{D})$ relationship for the crystal, to two related and quite similar double-sheeted surfaces: the wave-normal surface, relating the magnitude of wave velocity to direction, and the ray surface, playing the same role for rays. As an example of the pitfalls of the nomenclature, "Fresnel's wave surface" is in fact the ray surface.

Figure 4(a) shows a beautiful and rare (perhaps unique) wire model of the double-sheeted wave-normal surface (or alternatively the ray surface) for a biaxial crystal: this is the lowest possible optical symmetry. Figure 4(b) shows a plaster model of part of the outer sheet of the same surface. A radial line drawn in most directions will encounter two parts of the wave surface, the inverse radii giving the velocities of two possible waves, of different polarisation.

But two directions (together with their opposites) are very special: only a single velocity is found. In this direction the inner and outer surfaces intersect as in a diablo - two shallow cones touching point to point (figure 5). These special lines are called the optic axes of wave normals, or binormals (or several other names), in the case of the wave-normal surface.

At first sight these binormals might seem less interesting than the general directions that yield two distinct wave velocities and hence are associated with double refraction, but Hamilton saw that even more exotic refractive properties are associated with the optic axes.

So what kind of waves can be constructed having this very special choice of wave vector? It turns out that any perpendicular direction of $\mathbf{D}$ is acceptable and that as we rotate it around $\mathbf{k}$, the corresponding $\mathbf{E}$ vector describes a cone, as does $\mathbf{S}$ also. Recall that the Poynting vector $\mathbf{S}$ determines the direction of the corresponding rays. The rays associated with this special choice of wave vector are infinite in number and travel in all of the directions of a cone.
The ray surface has biradial lines like the binormals of the wavenormal surface. The biradial ray corresponds to waves whose directions are again disposed in a cone. The similarity of the two surfaces and close proximity of the directions of binormal and biradial lines is a further source of confusion that permeates the subject.

Conical refraction arises from the properties of these two kinds of special directions in the crystal. They require different experimental arrangements, as follows.

\section{Internal conical refraction}

Internal conical refraction is the case shown in figure 2(a). Its name is appropriate: the cone lies within the crystal. To set up an experimental demonstration, we must direct an incident beam so that a refracted wave would travel within the crystal in the direction of the binormal. So instead of one or two discrete rays, a diverging cone of rays fans out within the crystal. On leaving the back face they refract into a hollow cylindrical beam. That this final beam is perfectly circular is one of the strangest aspects of the effect; it is not related to any trivial symmetry. We have to resort to crystals of the lowest symmetry to produce this strikingly symmetric consequence! It goes against the grain of intuition.

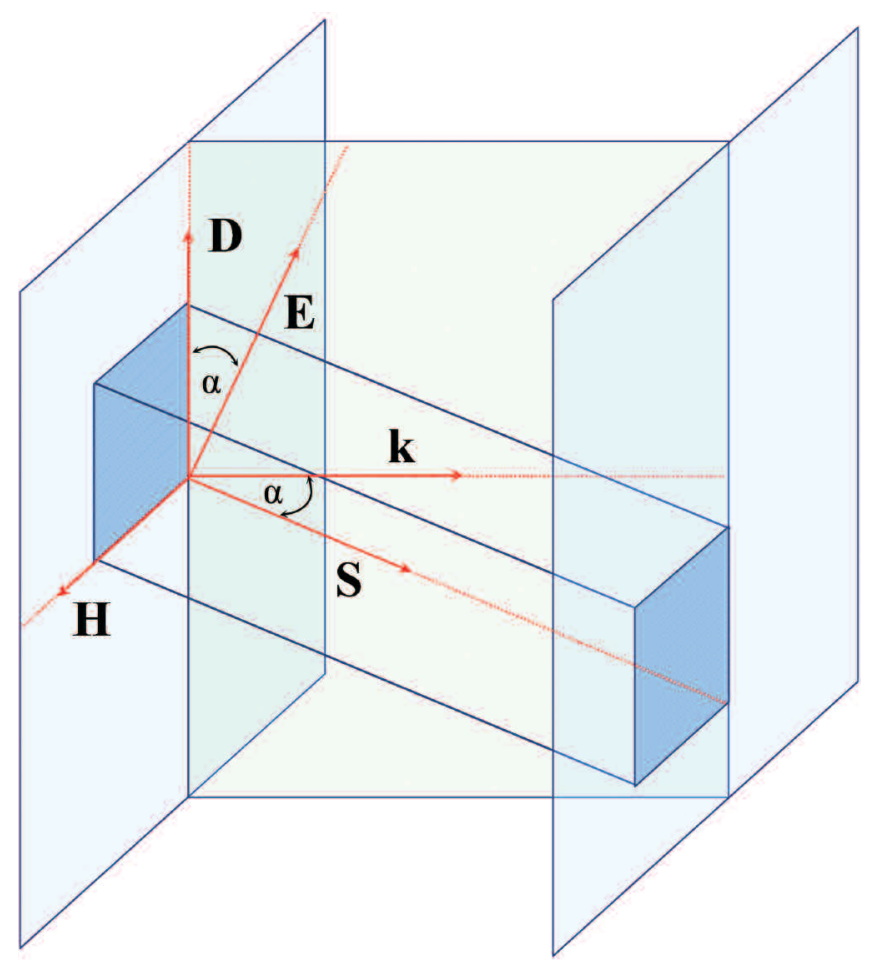

$\Delta$ Fig. 3: Relative orientations of the vectors $\mathbf{E}, \mathbf{D}, \mathbf{H}, \mathbf{k}$ and $\mathbf{S}$ associated with a plane electromagnetic wave (adapted from Light by Ditchburn [6]). 

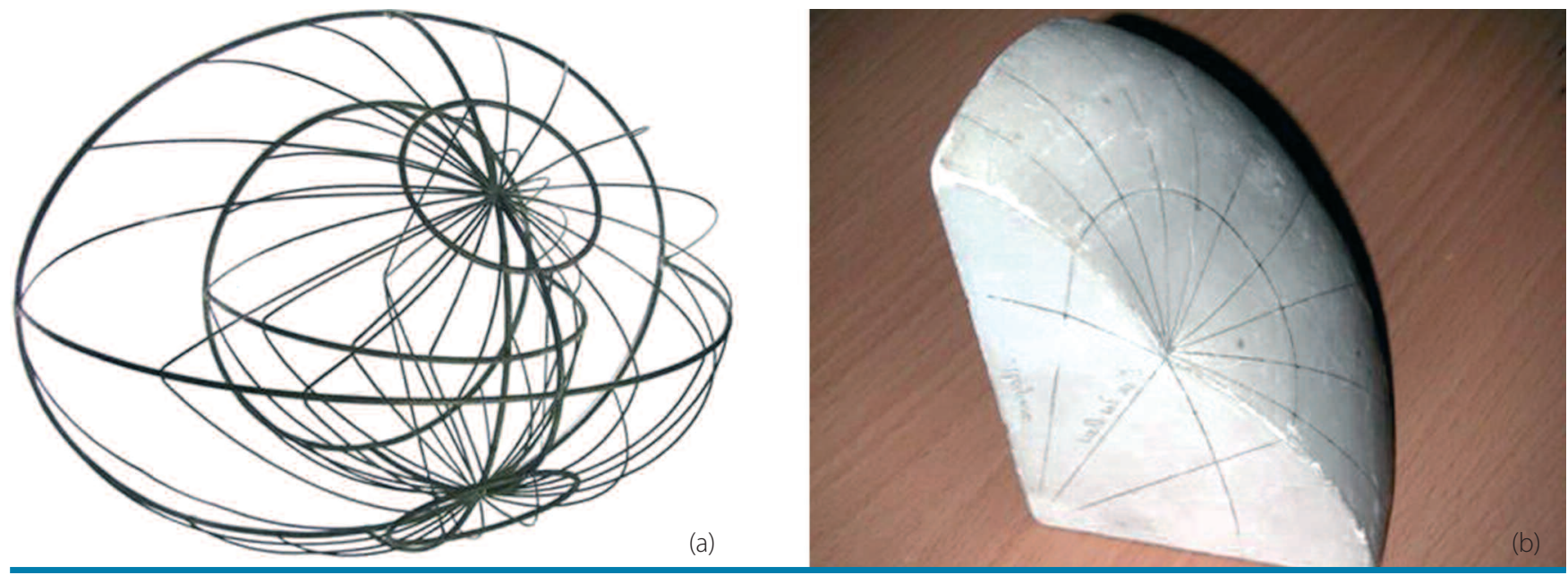

A Fig. 4: (a) Wire model of the double-sheeted wave-normal (or ray) surface of a biaxial crystal (courtesy of lan Sanders, Trinity College Dublin). (b) Plaster model of the outer sheet of the same surface (courtesy of Colin Latimer, Queen's University Belfast).

\section{External conical refraction}

Lloyd first looked for external conical refraction, which produces a diverging hollow cone of light. For this, a beam must travel through the crystal along a biradial line, rather than a binormal.

In the arrangement shown in figure 2(b), pinholes allow only propagation along the required direction, and a lens focuses light on the front pinhole, so that some of its light is refracted in the necessary direction. This ray can be considered as a mixture of waves, whose directions form a cone. They refract at the back face and a cone of rays emerges, each ray associated with a refracted wave. Thrown up on a screen to form a circular image, this is the radiant stranger of 1832 .

These experimental arrangements may today be simplified, if a crystal is cut so that its faces are normal to either the binormal or biradial directions. And lasers provide convenient bright beams, making demonstrations possible even on a large scale. Yuriy Mikhaylichenko of Tomsk University has made a speciality of this in recent years [7]. He has used crystals of sulphur in which the semiangle of conical refraction is quite large at about $3.5^{\circ}$.

\section{Hamilton's prediction}

Although only 27 at the time of this discovery, William Rowan Hamilton was already a senior and celebrated academic. He had been diverted into the position of professor of astronomy, directing Dunsink observatory some 5 miles from Trinity College and rejoicing in the title of Royal Astronomer of Ireland, while still an undergraduate. The college politics of that extraordinary appointment remain obscure, but it did give the young man an opportunity to fully immerse himself in his mathematics. Provided, that is, that he was not obliged to take observational astronomy too seriously.

+

His first intensive research programme was in optics, which he explored with characteristic rigour and originality. Out of that programme, in the fourth of a series of papers, came conical refraction in 1832. It was to become a favourite topic of correspondence between Hamilton and Peter Guthrie Tait (1831-1901) of Edinburgh, now available in a collection of their letters edited by David Wilkins [8].

\section{Lloyd's experiments}

In 1832 Hamilton's colleague Humphrey Lloyd had recently taken up the chair of natural and experimental philosophy at Trinity, following his father Bartholomew Lloyd in a distinguished career as an academic scientist and eventually as provost. His early career was devoted to optics, which he saw as a wonderful probe of the physics of materials, not just a set of rules for the propagation of light. So he eagerly grasped the opportunity to provide experimental confirmation for Hamilton.

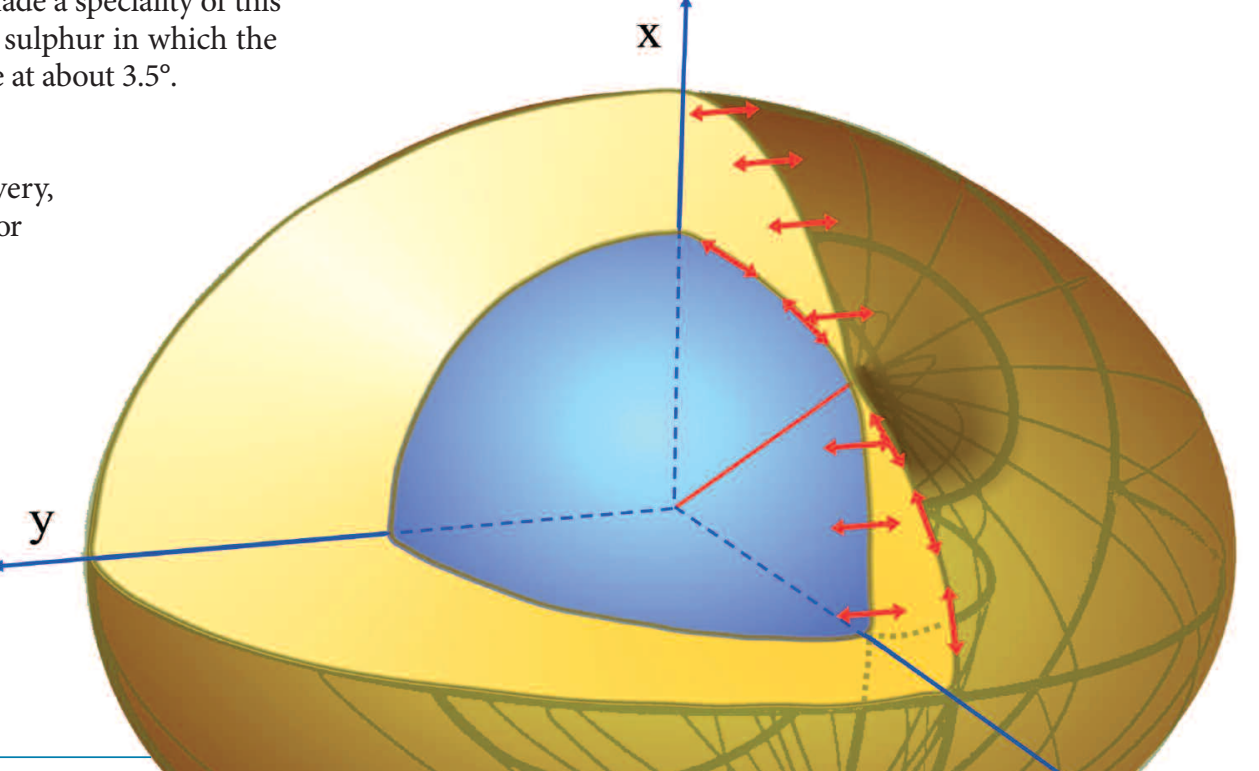

Fig. 5: Diagram of the double-sheeted wavenormal surface showing the intersection of the two sheets and one of the binormals. The polarisations of waves propagating in the $x-y$ plane are also shown. 
He had no success until he obtained a particularly good crystal of aragonite from a commercial supplier. With this he demonstrated external conical refraction, measuring a cone angle of about 3 degrees, as predicted. Lloyd was a thorough experimentalist, a fitting counterpart to Hamilton the systematic theorist. Not content with seeing the phenomenon as predicted, he examined the polarisation of the emergent light, and was surprised to find that "every ray of the cone was polarised in a different plane", as is shown in figure 6(b). Hamilton had passed over this obvious consequence of his theory.

Lloyd encountered much more difficulty when he tackled internal conical refraction, which is the favourite today, but he soon enjoyed the same success. He gave a full account of both experiments in the Proceedings of the Royal Irish Academy (1833), and a more public airing at the meeting of the British Association for the Advancement of Science (1834), on the same occasion that he presented his authoritative report on "the progress and present state of physical optics". It was a triumph for the tiny community of Dublin physicists, and may have influenced the decision to bring that Association's meeting to the city in the following year, in spite of some opposition. It must have been reckoned worth a rough sea crossing to an unruly country, to visit the home of Hamilton. It was at the meeting that, as reported by Robert Perceval Graves, "Lord Normanby, then Lord Lieutenant, seized, with a happy tact, the opportunity of paying a most handsome compliment both to Professor Hamilton and to Ireland, by conferring the foremost representative of our nation's science, and in the face of the assembled Association, with the honour of knighthood" [9].

\section{The finer details}

As early as 1839 , the bright ring exhibited by internal conical refraction was resolved by the experiments of Poggendorf into two concentric rings (Everything seems to come in twos in this subject). Figure 6(a) shows the distribution of intensity produced by internal conical refraction in a $2 \mathrm{~cm}$ long crystal of $\mathrm{KGd}\left(\mathrm{WO}_{4}\right)_{2}$. The same is true of the external case, though Lloyd did not observe this double ring structure, since the size of the optical apertures in his experiment caused the two circles to be blurred. With modern equipment, additional fainter rings are discernible and Lloyd's simple manifestation of the effect has become a complex pattern, offering a fresh challenge to theoreticians.

The extra structure appears because the light beams that are used are of finite extent, rather than those idealised rays, mere lines, upon which the elementary theory is based. The modern methodology of Gaussian (and related) laser beams can provide an adequate representation of the finite beam, and its propagation can be computed in full and compared with experiment [10 - 12].

\section{Conclusion}

Often in constructing (or deconstructing) the history of science, the story is edited for the sake of clarity. J. J. Thomson did not discover the electron on a certain day in Cambridge, but we tell it so. The case of conical refraction is an exception, in which the revelatory process is unambiguous, and priority is sharply defined - in spite of the anguished protestations of MacCullagh. It is a tale of two virtuous and dedicated scientists who richly deserved the accolades that they received. It is a tale worth recounting, for generations to come. The emergence of the radiant stranger can still startle, entertain and educate us. .

\section{About the authors}

James G. Lunney is Head of the School of Physics at Trinity College Dublin. He has worked on X-ray spectroscopy of laser produced plasmas for laser fusion. His current research is on laser ablation of solids and pulsed laser deposition of thin films for materials science research.

Denis Weaire, FRS is Erasmus Smith's Professor of Natural and Experimental Philosophy at Trinity College Dublin and is a former President of the EPS. In addition to research on the physics of foams, he has interests in the history of physics and currently serves as Chairman of its Interdivisional Group.

\section{Acknowledgements}

The authors would like to acknowledge helpful advice from Michael Berry on the preparation of this article and the work of Chris Smith in producing the illustrations.

\section{References}

[1] T. Preston, Theory of Light (Macmillian \& Co.) (1890).

[2] D.R.Wilkins, Physics World, August (2005).

[3] J O'Hara, Proceedings of Royal Irish Academy, 82A, 231, (1982).

[4] C.V. Raman, V.S. Rajagopalan and T.M.K. Nedungadi, Proc. Ind. Acad. Sci. A14, 221, (1941).

[5] M.V. Berry, Nature, 403, 21, (2000).

[6] R.W. Ditchburn, Light, (Academic Press) (1976).

[7] Y.P. Mikhaylichenko, Izv. Vuzov. Physika, No. 6, 96, (2000) [in Russian].

[8] D.R Wilkins, Perplexingly easy: selected correspondence between William Rowan Hamilton and Peter Guthrie Tait, (Trinity College Dublin Press and School of Physics, Trinity College Dublin) (2005).

[9] R.P. Graves, Dublin University Magazine, 19, 94, (1842).

[10] A.J. Schell and N. Bloembergen, J. Opt. Soc. Amer. 68, 1093, (1978).

[11] M.V. Berry, J. Optics A, 6, 289, (2004).

[12] M.V.Berry, M.R. Jeffery and J.G. Lunney, Proc. Roy. Soc. (to be published).
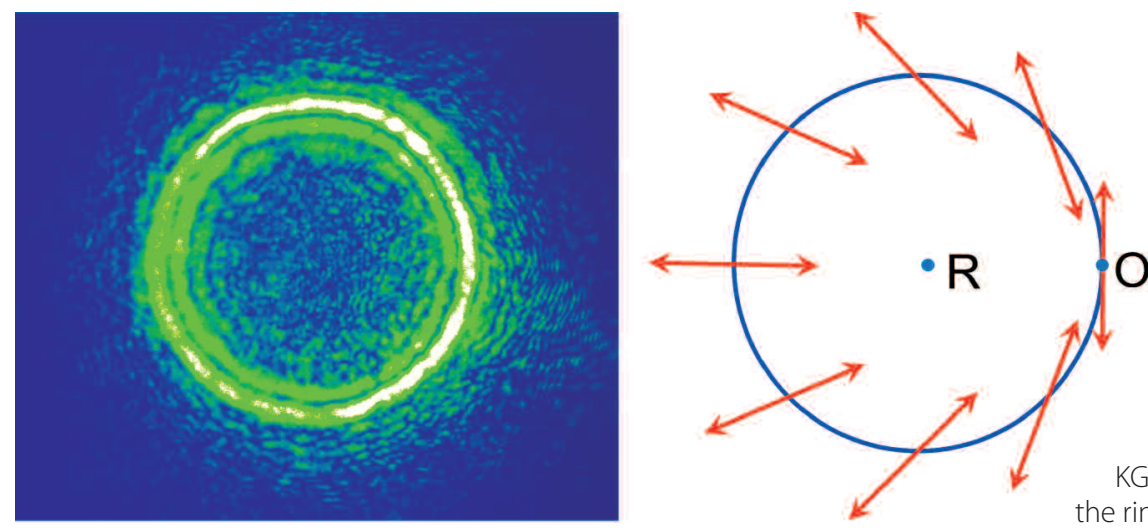

Fig. 6: (a) Beam profile produced by internal conical refraction of a $532 \mathrm{~nm}$ Gaussian laser beam in a $2 \mathrm{~cm}$ long crystal of $\mathrm{KGd}\left(\mathrm{WO}_{4}\right)_{2}$. (b) Variation of the polarisation around the ring-shaped light beam. 